\title{
STAR FORMATION IN AEGIS FIELD GALAXIES SINCE $z=1.1$ : THE DOMINANCE OF GRADUALLY DECLINING STAR FORMATION, AND THE MAIN SEQUENCE OF STAR-FORMING GALAXIES
}

\author{
K. G. Noeske, ${ }^{1}$ B. J. Weiner, ${ }^{2}$ S. M. Faber, ${ }^{1}$ C. Papovich, ${ }^{2}$ D. C. Koo, ${ }^{1}$ R. S. Somerville, ${ }^{3}$ K. Bundy, ${ }^{4}$ C. J. Conselice, ${ }^{5}$ \\ J. A. Newman, ${ }^{6,7}$ D. Schiminovich, ${ }^{8}$ E. Le Floc' ${ }^{2},{ }^{2}$ A. L. Coil,${ }^{2,7}$ G. H. Rieke, ${ }^{2}$ J. M. Lotz, ${ }^{9}$ J. R. Primack, ${ }^{10}$ \\ P. Barmby, ${ }^{11}$ M. C. Cooper, ${ }^{12}$ M. Davis, ${ }^{12}$ R. S. Ellis,${ }^{4}$ G. G. Fazio, ${ }^{11}$ P. Guhathakurta,${ }^{1}$ J. Huang, $^{11}$ \\ S. A. Kassin, ${ }^{1}$ D. C. Martin, ${ }^{4}$ A. C. Phillips, ${ }^{1}$ R. M. Rich, ${ }^{13}$ T. A. Small, ${ }^{4}$ \\ C. N. A. Willmer, ${ }^{2}$ AND G. Wilson ${ }^{14}$ \\ Received 2006 June 4; accepted 2007 January 31; published 2007 April 2
}

\begin{abstract}
We analyze star formation (SF) as a function of stellar mass $\left(M_{*}\right)$ and redshift $z$ in the All-Wavelength Extended Groth Strip International Survey. For 2905 field galaxies, complete to $10^{10}\left(10^{10.8}\right) M_{\odot}$ at $z<0.7(1)$, with Keck spectroscopic redshifts out to $z=1.1$, we compile SF rates (SFRs) from emission lines, GALEX, and Spitzer MIPS $24 \mu \mathrm{m}$ photometry, optical-NIR $M_{*}$ measurements, and HST morphologies. Galaxies with reliable signs of SF form a distinct "main sequence" (MS), with a limited range of SFRs at a given $M_{*}$ and $z$ ( $1 \sigma \lessgtr \pm 0.3$ dex), and $\log$ (SFR) approximately proportional to $\log M_{*}$. The range of $\log$ (SFR) remains constant to $z>1$, while the MS as a whole moves to higher SFR as $z$ increases. The range of the SFR along the MS constrains the amplitude of episodic variations of SF and the effect of mergers on the SFR. Typical galaxies spend $\sim 67 \%(95 \%)$ of their lifetime since $z=1$ within a factor of $\$ 2(4)$ of their average SFR at a given $M_{*}$ and $z$. The dominant mode of the evolution of SF since $z \sim 1$ is apparently a gradual decline of the average SFR in most individual galaxies, not a decreasing frequency of starburst episodes, or a decreasing factor by which SFRs are enhanced in starbursts. LIRGs at $z \sim 1$ seem to mostly reflect the high SFR typical for massive galaxies at that epoch. The smooth MS may reflect that the same set of few physical processes governs SF prior to additional quenching processes. A gradual process like gas exhaustion may play a dominant role.
\end{abstract}

Subject headings: galaxies: evolution — galaxies: formation — galaxies: high-redshift — galaxies: starburst

\section{INTRODUCTION}

Deep galaxy surveys have found consistently that the star formation rate (SFR) per unit stellar mass $\left(M_{*}\right)$ depends strongly on both $M_{*}$ and redshift $z$, with the bulk of star formation (SF) occurring earlier in massive galaxies than in less massive systems (e.g., Guzmán et al. 1997; Brinchmann \& Ellis 2000; Juneau et al. 2005; Bauer et al. 2005; Bell et al. 2005; Pérez-González et al. 2005; Feulner et al. 2005; Papovich et al. 2006; Caputi et al. 2006; Reddy et al. 2006). High-SFR objects are observed to be more abundant at higher $z$; it is often assumed that a part of these reflect a greater frequency of merger-driven starburst episodes at earlier times. However, a comprehensive observational picture of the relationship between SF and mass to $z \sim 1$, including objects with a wide range of both masses and SF rates, has been lacking.

This Letter is part of a series of papers that study the evo-

${ }^{1}$ UCO/Lick Observatory, University of California, Santa Cruz, CA 95064.

${ }^{2}$ Steward Observatory, University of Arizona, Tucson, AZ 85721.

${ }^{3}$ Max-Planck-Institut für Astronomie, 69117 Heidelberg, Germany.

${ }^{4}$ California Institute of Technology, Pasadena, CA 91125.

${ }^{5}$ School of Physics and Astronomy, University of Nottingham, Nottingham NG9 2RD, UK.

${ }^{6}$ Institute for Nuclear and Particle Astrophysics, Lawrence Berkeley National Laboratory, Berkeley, CA 94720.

${ }^{7}$ Hubble Fellow.

${ }^{8}$ Department of Astronomy, Columbia University, New York, NY 10027.

${ }^{9}$ Leo Goldberg Fellow, National Optical Astronomy Observatory, Tucson, AZ 85718.

${ }^{10}$ Department of Physics, University of California, Santa Cruz, CA 95064

${ }^{11}$ Harvard-Smithsonian Center for Astrophysics, Cambridge, MA 02138.

${ }^{12}$ Department of Astronomy, University of California, Berkeley, CA 94720.

${ }^{13}$ Department of Physics and Astronomy, University of California, Los Angeles, CA 90095-1547.

${ }^{14}$ Spitzer Science Center, California Institute of Technology, Pasadena, CA 91125 . lution of the SFR and $M_{*}$ in field galaxies out to $z=1.1$ in the All-Wavelength Extended Groth Strip International Survey (AEGIS). We combine SFR measurements from deep Multiband Imaging Photometer for Spitzer (MIPS) $24 \mu \mathrm{m}$ imaging, Keck/DEEP2 spectra, and Galaxy Evolution Explorer (GALEX) UV photometry, allowing us both to recover obscured SF in IR-luminous galaxies and to include lower SFR objects not detected at $24 \mu \mathrm{m}$. Using optical-near-IR derived $M_{*}$ measurements, we analyze the evolution of the SFR as a function of $M_{*}$ and $z$; we also analyze Hubble Space Telescope (HST) Advanced Camera for Surveys imaging and rest-frame colors to support the interpretation of SFR indicators.

We adopt a concordance cosmology $\left(H_{0}=70 \mathrm{~km} \mathrm{~s}^{-1} \mathrm{Mpc}^{-1}\right.$, $\left.\Omega_{M}=0.3, \Omega_{\Lambda}=0.7\right)$. Values of $M_{*}$ and SFR are based on a Kroupa (2001) initial mass function, following recent results by Hopkins \& Beacom (2006).

\section{DATA SET}

Our sample includes all field galaxies with DEEP2 spectroscopic redshifts $z \leq 1.1$, in the area where Spitzer MIPS $24 \mu \mathrm{m}$ photometry and $K$-band imaging to $22 \mathrm{AB}$ mag are available; (see Davis et al. 2007). Stellar masses were obtained from spectral energy distribution (SED) fits to optical/NIR photometry by Bundy et al. (2006); errors are $<0.3$ dex, with a mean and rms of 0.1 and 0.05 dex and $<4 \%$ of errors $>0.2$ dex.

Figure 1 shows data in the $M_{*}$ range where the sample is $>80 \%$ complete, adopting the completeness analysis by Bundy et al. (2006; see also Cimatti et al. 2006), for a total of 2905 galaxies. We draw conclusions only where the sample is $>95 \%$ complete $\left[M_{*} \geq 10(10.8)\right.$ dex $M_{\odot}$ for $z<0.7(1)$; see vertical lines in Fig. 1]. For galaxies with robust $24 \mu \mathrm{m}$ detections $(f>60 \mu \mathrm{Jy})$, SFRs were derived following Le Floc'h et al. 


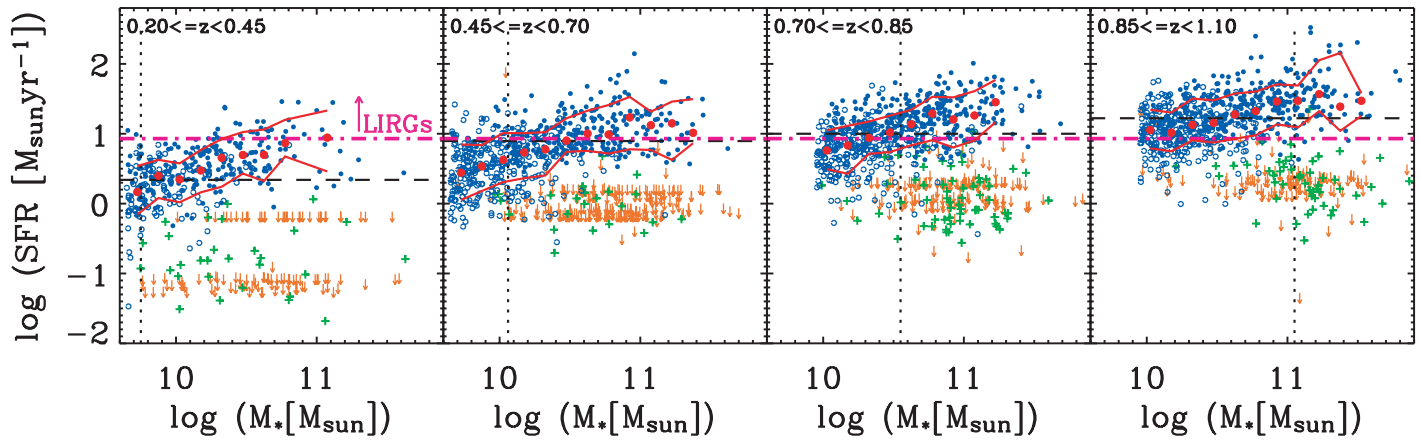

FIG. 1.-SFR vs. $M_{*}$ for 2905 galaxies in the Extended Groth Strip, in the $M_{*}$ range where the data are $>80 \%$ complete; see $\S 2$. The dotted vertical line marks $>95 \%$ completeness. Filled blue circles: Combined SFRs from MIPS $24 \mu \mathrm{m}$ and DEEP2 emission lines. Open blue circles: No $24 \mu \mathrm{m}$ detection, blue $U-B$ colors, SFR from extinction-corrected emission lines. Green plus signs: Same as open blue circles, but red $U-B$ colors, mostly LINER/AGN candidates ( $\$ 3$ ). Orange downward arrows: No robust detection of $f(24 \mu \mathrm{m})$ or emission lines; conservative SFR upper limits shown. There is a distinct sequence formed by fiducial SF galaxies (open and filled circles); galaxies with little or no SF lie below this sequence. Red circles show the median of log (SFR) in mass bins of 0.15 dex for MS galaxies (blue circles). Red lines include 34\% of the MS galaxies above and 34\% below the median of log (SFR), $\pm 1 \sigma$ in the case of a normal distribution. Horizontal black dashed line: SFR corresponding to the $24 \mu \mathrm{m} 80 \%$ completeness limit at the center of each $z$ bin; $24 \mu \mathrm{m}-\mathrm{detected}$ galaxies above the magenta dot-dashed line are LIRGs (§ 4.2).

(2005), using Chary \& Elbaz (2001) SED templates; using templates from Dale \& Helou (2002) yields no significant differences. We then add to the $24 \mu \mathrm{m}$-based SFR the SFR estimated from DEEP2 emission lines $(\mathrm{H} \alpha, \mathrm{H} \beta$, or [O II] $\lambda 3727$, depending on $z$ ) with no extinction correction, to account for $\mathrm{SF}$ from unobscured regions. This approach is similar to that employed by Bell et al. (2005); utilizing rest-frame UV continuum SFRs (as they did) in place of emission-line fluxes yields consistent results. Galaxies below the $24 \mu \mathrm{m}$ detection limit are not dominated by highly extincted SF; for these, we use extinction-corrected SFRs from emission lines; these can probe to roughly 10 times lower SFRs than the $24 \mu \mathrm{m}$ data and are slightly more sensitive at high $z$ and cover a larger area than GALEX data. Emission-line luminosities (as calculated in Weiner et al. 2006) were transformed to an $\mathrm{H} \alpha \mathrm{lu}-$ minosity using average line ratios measured from DEEP2 data $(\mathrm{H} \beta / \mathrm{H} \alpha=0.198$, [O II] $/ \mathrm{H} \alpha=0.69$; B. J. Weiner 2006, private communication) and transformed to the SFRs using the $\mathrm{H} \alpha$ calibration of Kennicutt (1998). The DEEP $2 \mathrm{H} \beta / \mathrm{H} \alpha$ ratio corresponds to an extinction of $1.30 \mathrm{mag}$ at $\mathrm{H} \alpha$ assuming case $\mathrm{B}$ recombination, which was applied to correct the emissionline SFRs. We use fixed rather than $M_{\mathrm{H}}$-dependent line ratios (à la Weiner et al.), because these predict extinction-corrected SFRs slightly in excess of the $24 \mu \mathrm{m}$-derived SFRs for highmass galaxies. Our simple but robust approach yields results in good agreement with SFRs derived from GALEX data, extinction-corrected based on UV spectral slopes.

For objects with both $f_{24 \mu \mathrm{m}}<60 \mu \mathrm{Jy}$ and emission-line signalto-noise ratio $(\mathrm{S} / \mathrm{N})<2$, we estimate a $2 \sigma$ upper limit on SFRs from the most sensitive emission line available, by adding $2 \sigma$ to the measured uncertain SFR, or, for nondetections, to the limit of S/N > 2-detectable emission-line SFRs at the galaxy's redshift. We again apply $A_{\mathrm{H} \alpha}=1.30$ for extinction corrections, certainly an overestimate since extinction is lower in more weakly star-forming galaxies (Hopkins et al. 2001).

We have performed a suite of tests of these SF estimates, finding that adopting different SFR tracers changes results moderately (K. G. Noeske et al. 2007, in preparation); qualitative results are unaffected. Random errors in our $24 \mu \mathrm{m}$-based SFRs are $\lesssim 0.1$ dex from photometry and $\sim 0.15$ dex from scatter in the $f(24 \mu \mathrm{m})$ to $L(\mathrm{IR})$ conversion (see Marcillac et al. 2006), yet total random errors are expected to be 0.3-0.4 dex (see Bell et al. 2005). For extinction-corrected emission-line SFRs, random errors are $\sim 0.35$ dex, including scatter about the assumed mean extinction.

\section{RESULTS}

Figure 1 shows the SFR as a function of $M_{*}$ in four independent redshift bins. The following discussion refers only to the stellar mass range where the sample is $>95 \%$ complete, marked by the vertical dotted lines in each redshift bin. We identify three different categories of galaxies:

1. The majority of galaxies show clear signs of SF, either robust $24 \mu \mathrm{m}$ detections or, at lower $M_{*}$, blue colors and emission lines (blue symbols in Fig. 1). Quantitative HST morphologies (Gini/M20: Lotz et al. 2007; CAS: Conselice 2003) classify $\lesssim 25 \%$ of these galaxies as early types (E, S0, Sa), and $\gtrsim 90 \%$ show visual signs of SF such as blue regions and dust lanes. Most of them lie on the "blue cloud" (e.g., Willmer et al. 2006), although some of the massive ones are red, likely dusty, star-forming galaxies (Bell et al. 2005). This category (blue symbols in Fig. 1) comprises 67\%(56\%) of the sample at $z<(>) 0.7$ in the $M_{*}$ range where the sample is complete.

2. Clearly separated are galaxies without robust $24 \mu \mathrm{m}$ ( $>60 \mu \mathrm{Jy}$ ) or emission-line $(\mathrm{S} / \mathrm{N}>2$ ) detections (orange arrows in Fig. 1). The upper limits on their SFRs are conservatively high ( $\$ 2)$, such that the true separation between the sequence and the other galaxies is likely larger than it appears here. Almost all $(>95 \%)$ of these galaxies are on the red sequence, and $\gtrsim 90 \%(80 \%)$ at $z<(>) 0.7$ have early-type quantitative morphologies including early-type mergers, while $\gtrsim 90 \%$ at $z>$ 0.7 have early-type visual morphologies with no hints of current SF. These galaxies contribute $29 \%(30 \%)$ of the sample at $z<(>) 0.7$.

3. Scattered below the star-forming sequence are galaxies with robust emission-line detections but no significant $24 \mu \mathrm{m}$ emission, $5 \%(14 \%)$ of the sample at $z<(>) 0.7$. All of these galaxies (green plus signs in Fig. 1) are on the red sequence, and their $\mathrm{H} \alpha, \mathrm{H} \beta$ emission-line equivalent widths tend to be low (a few angstroms). Yan et al. (2006) and Weiner et al. (2006) showed that the bulk of the line emission in red galaxies out to intermediate redshifts is due to LINER/AGN emission, not SF. We find that $75 \%$ of those galaxies with $[\mathrm{O}$ II] and $\mathrm{H} \beta$ detections show LINER-like line ratios, and $\gtrsim 55 \%(70 \%)$ at $z<(>) 0.7$ have early-type quantitative and visual morphologies that are typical 
for local LINERs (Yan et al. 2006). Line emission in these red galaxies thus appears to be dominated by LINERs/AGNs, particularly at $z>0.7$ where they are more frequent. Their SFRs, derived from emission lines (Fig. 1), will mostly be overestimated. However, we find visual signs of SF in the HST images in $\$ 30 \%$ of these galaxies, comparable to the fraction of nonearly quantitative morphologies; these may be dominated by SF.

The star-forming galaxies form a distinct sequence of SFRs with $M_{*}$, which we term the "main sequence" (MS). The red lines in Figure 1 enclose $34 \%$ of galaxies both above and below the median (red circles) and thus indicate the equivalent of $\pm 1 \sigma$ for a Gaussian distribution. The width of the MS measured in this way (the range in SFR about the median at a given $M_{*}$ ) is about $\sigma_{\mathrm{MS}}=0.35 \mathrm{dex}$ and seems to remain approximately constant in our sample over the redshift range $0.20<z<1.1$. Subtracting a lower limit of nonsystematic scatter in SFR $(\sim 0.2$ dex; $\S 2)$ in quadrature yields an upper limit of $\sim 0.3 \mathrm{dex}$ on the intrinsic scatter, which is still broadened by the width of the $z$ bins, and by additional spread from combining different SFR tracers. Errors in $M_{*}$ hardly affect $\sigma_{\mathrm{MS}}$.

The slope of the MS is shallower than unity, $\log (\mathrm{SFR})=$ $(0.67 \pm 0.08) \log M_{*}-(6.19 \pm 0.78)$, for $M_{*}$ between $10^{10}$ and $10^{11} M_{\odot}$, and $z=0.2-0.7$. There is a trend for the slope to flatten to higher $z$, but the completeness limits do not allow a robust quantification. A further important result is that the normalization of the MS evolves strongly over the redshift range of our sample; the median SFR at fixed $M_{*}$ evolves downward by a factor of 3 , measured at $10^{11} M_{\odot}$, from the our highest (median $z=0.98$ ) to our lowest (median $z=$ 0.36) redshift bin. Importantly, it appears that the whole of the $M S$ shifts downward with time, rather than just the upper envelope decreasing, which was also reported by a recent GALEX study at $z=0.7$ (Zamojski et al. 2007). A straightforward interpretation of these observations is that normal star-forming galaxies possess a limited range of SFRs at a given $M_{*}$ and $z$, which is presumably set by whatever physical processes regulate SF in quiescent disks. Galaxies that are not on the MS, in categories 2 and 3 above, are observed during or after quenching of the SF activity, with either low-level or no current SF, or LINER/AGN activity.

\section{DISCUSSION}

\subsection{Completeness: Is the Main Sequence Real?}

It is obviously crucial to determine whether the MS that we have identified is real or could be caused by selection effects or observational biases. We address the following possible causes of incompleteness or bias in our sample, again restricting the discussion to the $M_{*}$ range where we claim that the sample is $>95 \%$ complete: (1) Could the optically selected DEEP2 parent sample be missing a significant number of galaxies, or are there galaxies in the DEEP2 sample that lack a successful redshift determination because of low S/N? (2) Could we be significantly underestimating the SFRs in galaxies in our sample due to biases in our SF indicators?

1. The DEEP2 spectroscopic selection $\left(R_{\mathrm{AB}}<24.1\right)$ has been shown to be complete in the $M_{*}$ ranges indicated in Figure 1 (vertical lines), from comparisons to various surveys with spectroscopic and deep photometric redshifts, including in particular the $K$-selected K20 survey, which should be less affected by extinction (Willmer et al. 2006; Bundy et al. 2006; Cimatti et al. 2006). For galaxies that are below our $24 \mu \mathrm{m}$ detection limit, we expect the extinction to be moderate and would expect these galaxies to be picked up in $K$-selected surveys, but no such population is found to be missed by DEEP2.

More obscured populations can be probed through the deep Spitzer Infrared Array Camera (IRAC) $3.6 \mu \mathrm{m}$ data in AEGIS, which at a given redshift are a proxy for $M_{*}$ yet are barely affected by extinction. We have compared the distribution of $f(24 \mu \mathrm{m})$ at a given $f(3.6 \mu \mathrm{m})$ and $z$ in the DEEP2 $R_{\mathrm{AB}}$-selected sample and an IRAC $f(3.6 \mu \mathrm{m})$-selected sample with IRACbased photometric redshifts. We find no evidence that DEEP2 misses a significant population of heavily obscured, starforming galaxies at $z \lesssim 1$, which could populate the area above the upper boundary of the MS. This agrees with the results of Houck et al. (2005) and Weedman et al. (2006) in the largearea NOAO Deep Wide-Field Survey, which indicate that such missed $f(24 \mu \mathrm{m})$-bright, optically faint galaxies at $z<1$ would contribute $<1 \%$ of our sample.

2. The $24 \mu \mathrm{m}$ completeness limit (horizontal black dashed line in Fig. 1) intersects the MS in each redshift bin. As discussed in $\S 3$, most galaxies below the MS are red, early type, non-SF, and/or LINER/AGN-dominated (shown as orange arrows and green plus signs). However, a fraction show spiral/late-type morphologies or visual signs of possible SF ( $\$ 3)$. In principle, these red galaxies could have dust-obscured SF, unrecovered by emission lines, yet lie below the $24 \mu \mathrm{m}$ detection limit. Their true SFRs could then be anywhere up to the $24 \mu \mathrm{m}$ limit, in which case they may not be a distinct population but rather a downward continuation of the MS. If this were the case, these galaxies would make up $\leqslant 10 \%(20 \%)$ of the MS at $z<(>) 0.7$. We can constrain the maximal effect of missed, dust-obscured SF in these galaxies on the $1 \sigma$ range of SFRs along the MS by including in the calculation of $\sigma_{\mathrm{MS}}$ all red, $24 \mu \mathrm{m}$-undetected galaxies with spiral/late-type morphologies, and $\mathrm{H} \alpha, \mathrm{H} \beta$, and/or [O II] line emission down to spurious detections (i.e., $100 \%$ error in equivalent width). For the extremes of either only the emission line SFR or the maximal SFR, corresponding to the $24 \mu \mathrm{m}$ limit, the measured width of the MS increases by $\sim 0.05$ dex or not at all, respectively.

Thus, we argue that the relatively sharp upper limit of the MS is real, as our selection does not miss obscured sources with high SFRs. The sharpness of the lower limit is more uncertain with our current data, but we find that only a small fraction of galaxies that we placed below the MS could have underestimated SF rates that would "blur out" the lower edge of the MS. Very deep $24 \mu \mathrm{m}$ data at $z \sim 1$ from the Great Observatories Origins Deep Survey (D. Elbaz 2006, private communication) unambiguously confirm this result, particularly a well-defined lower boundary to the sequence.

\subsection{Constraints on Episodic Star Formation}

Studies based on local samples (Brinchmann et al. 2004; Salim et al. 2005 for the Sloan Digital Sky Survey; Lee 2006) have illustrated a relationship between the SFR and $M_{*}$ and have identified two populations: galaxies on a star-forming sequence and "quenched" galaxies with little or no detectable SF. At higher $z$, previous studies (see $\S 1$ ) had merely described an upper envelope of SFRs in the SFR- $M_{*}$ diagrams. We have employed a variety of SFR tracers and other evidence from AEGIS to show that the SF sequence persists out to $z \sim 1$, with a similar dispersion in $\log (\mathrm{SFR})$ at fixed $M_{*}$ but with a decrease in normalization of a factor of 3 from $z=0.98$ to $z=0.36$, measured at $M_{*}=10^{11} M_{\odot}$. The global SFR density has also decreased by a factor of 3 over this same interval (Hopkins 2004, eq. [3]). One possible physical explanation for this de- 
cline is a decreasing contribution from starbursts in gas-rich galaxy mergers. However, if this were the dominant factor causing the decline, we would expect to see the upper envelope of the MS move downward with time, with the region populated by "normal" galaxies maintaining the same normalization. This is contrary to what we see in AEGIS: the region of the SFR$M_{*}$ space populated by MS galaxies at $z \sim 0$ (Brinchmann et al. 2004) is nearly empty at $z \sim 0.7-1$, although these galaxies should be detectable in our survey.

We can use our observed MS to quantitatively constrain the duty cycle of episodic variations of SFRs around an average level. We adopt the densely populated peak of the SFR distribution (the median) as this baseline level. The 1(2) $\sigma$ ranges about the median of $\log (\mathrm{SFR}), \pm 0.3(0.6)$ dex, include $68 \%(95 \%)$ of the galaxies. We can hence infer that SFR variations exceeding $\pm 0.3(0.6)$ dex, factors of 2(4), have duty cycles $<32 \%(5 \%)$. These correspond to total times of $<2.5(0.4)$ Gyr since $z=1$. The amplitude of these variations $(\lessgtr \times 4)$ is consistent with gas-poor or minor mergers, rather than the peak SFR of gas-rich major mergers (Springel 2000; Cox et al. 2006). Excursions in SFR $>5$ times above the median are rare, $\sim 1 \%$, consistent with galaxies spending $\$ 100$ Myr in such strong burst episodes since $z=1$. Of course these arguments are only valid in a statistical sense: a fraction of galaxies could have a lower average level of SF and undergo larger excursions but at the expense of reducing the allowed range of SFRs for the remainder of the population.

Previous studies have found that $\$ 30 \%$ of SF at $z \sim 0.7$ occurs in morphologically disturbed galaxies (Wolf et al. 2005; Bell et al. 2005) or close pairs (Lin et al. 2007). Semianalytic models predict that about $5 \%$ of the $\mathrm{SF}$ at $z \sim 0.7$ is due to major mergers, with the contribution due to minor mergers being more uncertain but ranging from $\sim 11 \%$ to $45 \%$ (Somerville et al. 2001; Wolf et al. 2005). These direct constraints and theoretical expectations are consistent with the conclusions that we have drawn here from the SFR- $M_{*}$ distributions. A related comment pertains to the nature of luminous infrared galaxies [LIRGs; $L(8-1000 \mu \mathrm{m})>10^{11} L_{\odot}$ ]. LIRGs at $z \sim 0$ are rare, mostly interacting galaxies (Sanders \& Mirabel 1996) with strong starbursts (SFR $\gtrsim 5$ times above those of typical spirals). At $z \sim 1$, LIRGs seem to mostly represent the high level of SFRs in almost all massive SF galaxies, rather then extreme starbursts (Fig. 1).

In summary, we suggest a picture in which we are witnessing a gradual decline in the SFR of most galaxies since $z \sim 1$, accompanied by rapid quenching in a fraction of (massive) galaxies. Presumably, the regularity and constant dispersion of the MS out to $z \sim 1$ means that the same physics that regulates $\mathrm{SF}$ in local disk galaxies is operating, indicating significant evolution either in the gas supply or SF efficiencies over this interval.

In the accompanying Letter (Noeske et al. 2007), we show that the slope and evolution of the MS can be understood as gradual gas exhaustion in a model in which galaxy age and SF timescale are a function of galaxy mass, and the dispersion of the MS is interpreted as resulting from a spread in age and SF timescales at a given mass.

See the survey summary Letter (Davis et al. 2007) for full acknowledgments. This work is based on observations with the W. M. Keck Telescope, the Hubble Space Telescope, the Galaxy Evolution Explorer, the Canada-France-Hawaii Telescope, and the Palomar Observatory, and was supported by NASA and NSF grants. We wish to recognize the cultural role that the summit of Mauna Kea has within the Hawaiian community. This work is based in part on observations made with the Spitzer Space Telescope, which is operated by the Jet Propulsion Laboratory, California Institute of Technology, under a contract with NASA. Support for this work was provided by NASA through contract numbers 1256790, 960785, and 1255094 issued by JPL/Caltech. We wish to thank the referee for very valuable comments, and D. Elbaz and J. Lee for helpful discussions. K. G. N. acknowledges support from the Aspen Center for Physics.

\section{REFERENCES}

Bauer, A. E., Drory, N., Hill, G. J., \& Feulner, G. 2005, ApJ, 621, L89

Bell, E. F., et al. 2005, ApJ, 625, 23

Brinchmann, J., Charlot, S., White, S. D. M., Tremonti, C., Kauffmann, G., Heckman, T., \& Brinkmann, J. 2004, MNRAS, 351, 1151

Brinchmann, J., \& Ellis, R. S. 2000, ApJ, 536, L77

Bundy, K., et al. 2006, ApJ, 651, 120

Caputi, K. I., et al. 2006, ApJ, 637, 727

Chary, R., \& Elbaz, D. 2001, ApJ, 556, 562

Cimatti, A., Daddi, E., \& Renzini, A. 2006, A\&A, 453, L29

Conselice, C. J. 2003, ApJS, 147, 1

Cox, T. J., Jonsson, P., Primack, J. R., \& Somerville, R. S. 2006, MNRAS, 373, 1013

Dale, D. A., \& Helou, G. 2002, ApJ, 576, 159

Davis, M., et al. 2007, ApJ, 660, L1

Feulner, G., Gabasch, A., Salvato, M., Drory, N., Hopp, U., \& Bender, R. 2005, ApJ, 633, L9

Guzmán, R., Gallego, J., Koo, D. C., Phillips, A. C., Lowenthal, J. D., Faber, S. M., Illingworth, G. D., \& Vogt, N. P. 1997, ApJ, 489, 559

Hopkins, A. M. 2004, ApJ, 615, 209

Hopkins, A. M., \& Beacom, J. F. 2006, ApJ, 651, 142

Hopkins, A. M., Connolly, A. J., Haarsma, D. B., \& Cram, L. E. 2001, AJ, 122,288

Houck, J. R., et al. 2005, ApJ, 622, L105

Juneau, S., et al. 2005, ApJ, 619, L135
Kennicutt, R. C., Jr. 1998, ARA\&A, 36, 189

Kroupa, P. 2001, MNRAS, 322, 231

Lee, J. C. 2006, Ph.D. thesis, Univ. Arizona

Le Floc'h, E., et al. 2005, ApJ, 632, 169

Lin, L., et al. 2007, ApJ, 660, L51

Lotz, J. M., et al. 2007, ApJ, submitted (astro-ph/0602088)

Marcillac, D., Elbaz, D., Chary, R. R., Dickinson, M., Galliano, F., \& Morrison, G. 2006, A\&A, 451, 57

Noeske, K. G., et al. 2007, ApJ, 660, L47

Papovich, C., et al. 2006, ApJ, 640, 92

Pérez-González, P. G., et al. 2005, ApJ, 630, 82

Reddy, N. A., Steidel, C. C., Fadda, D., Yan, L., Pettini, M., Shapley, A. E., Erb, D. K., \& Adelberger, K. L. 2006, ApJ, 644, 792

Salim, S., et al. 2005, ApJ, 619, L39

Sanders, D. B., \& Mirabel, I. F. 1996, ARA\&A, 34, 749

Somerville, R. S., Primack, J. R., \& Faber, S. M. 2001, MNRAS, 320, 504

Springel, V. 2000, MNRAS, 312, 859

Weedman, D., et al. 2006, ApJ, 651, 101

Weiner, B. J., et al. 2007, ApJ, 660, L39

Willmer, C. N. A., et al. 2006, ApJ, 647, 853

Wolf, C., et al. 2005, ApJ, 630, 771

Yan, R., Newman, J. A., Faber, S. M., Konidaris, N., Koo, D., \& Davis, M. 2006, ApJ, 648, 281

Zamojski, M. A., et al. 2007, ApJS, in press (astro-ph/0701478) 